\title{
Adenovirus-mediated TA-p73 $\beta$ gene transfer increases chemosensitivity of human malignant melanomas
}

\author{
S. Tuve, T. Racek, A. Niemetz, J. Schultz, M. S. Soengas and B. M. Pützer \\ Department of Vectorology and Experimental Gene Therapy, University of Rostock, Schillingallee 70, 18055 Rostock, \\ Germany (S. Tuve, T. Racek, A. Niemetz, J. Schultz, B. M. Pützer); Department of Dermatology, University of Michigan \\ Comprehensive Cancer Center, Ann Arbor, MI 28109, USA (M. S. Soengas)
}

Published online: 18 February 2006

Malignant melanoma is the most aggressive form of skin cancer and has proven to be highly resistant to conventional chemotherapy. Intriguingly, the p53 tumor suppressor, a main mediator of chemoresistance in other tumor types, is rarely mutated in melanoma. However, we have previously shown that anti-apoptotic isoforms of p73 ( $\Delta$ TAp73), another member of the p53 family, are overexpressed in metastatic melanomas. $\triangle T A-p 73$ can oppose the proapoptotic functions of p53 and full length p73, and thus it could contribute to melanoma chemoresistance. In this study, we use an efficient adenoviral-based gene transfer approach to introduce a transcriptionally active form of p73 $(\mathrm{TA}-\mathrm{p} 73 \beta)$ in melanoma cells, with the objective of overcoming drug resistance. Interestingly, TA-p73 $\beta$ significantly sensitized 5 out of 7 aggressive melanoma cell lines to the standard therapeutic agents adriamycin and cisplatin. More importantly, TA-p73 $\beta$ displayed a synergistic effect in vivo allowing adriamycin or cisplatin to block melanoma cell growth in mouse xenograft models $(p<0.05)$. In summary, our data show that Ad-mediated TA-p73 $\beta$ gene expression can markedly sensitize a subset of melanoma cell lines to adriamycin and cisplatin in vitro and in vivo, suggesting a new chemosensitization strategy for malignant melanomas.

Keywords: apoptosis; chemotherapy; gene therapy; melanoma; p73.

\section{Introduction}

Malignant melanoma has been one of the fastest rising malignancies in the last decades, with a life time risk currently of $1 / 53$. This highly aggressive form of skin cancer is curable when resected at an early stage. In its metastatic stage, melanoma has an extremely poor prognosis since chemotherapy is ineffective due in part to the intrinsic and/or extrinsic resistance of melanoma cells to systemic treatment with anti-neoplastic agents. ${ }^{1}$ Most anticancer drugs kill susceptible cells through the induction of apoptosis. Therefore alterations of apoptotic pathways leading to apoptosis defi-

Correspondence to: B. M. Pützer, Department of Vectorology and Experimental Gene Therapy, University of Rostock, Schillingallee 70, D-18055 Rostock, Germany. Tel.: +49-381-494-5066; Fax: +49381-494-5062; e-mail: brigitte.puetzer@med.uni-rostock.de ciency represent a potent mechanism conferring drug resistance on melanoma cells. ${ }^{2}$ Acquired apoptotic defects during melanoma progression involve activation of anti-apoptotic factors, e.g. survivin, ${ }^{3}$ inactivation of proapoptotic factors, e.g. Apaf- $-1,{ }^{4}$ and upregulation of survival signals, e.g. B-

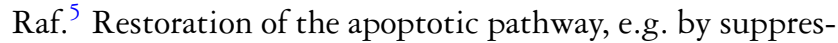
sion of survivin ${ }^{3}$ or activation of Apaf- $1,{ }^{4}$ has been shown to increase the sensitivity of melanoma cells to chemotherapeutic agents. As an important molecular component of apoptosis p53 influences the resistance to chemotherapy in malignant tumors. However, melanomas show a low frequency of p53 mutations despite their extreme chemoresistance. ${ }^{6}$ One explanation of this paradox is that disruption of pathways upstream and/or downstream of $\mathrm{p} 53$ could functionally replace $\mathrm{p} 53$ loss during melanomagenesis, e.g. inactivation of p14ARF ${ }^{7}$ or Apaf- $1 .{ }^{4}$

The $\mathrm{p} 53$ family member $\mathrm{p} 73$ shares significant sequence homology with $\mathrm{p} 53$. However, in contrast to $\mathrm{p} 53$, $\mathrm{p} 73$ gives rise to multiple protein isoforms due to alternative promoter utilization and alternative mRNA splicing. ${ }^{8}$ The TA isoforms of $\mathrm{p} 73$, e.g. TA-p $73 \alpha$ and TA-p $73 \beta$, contain the $\mathrm{N}$-terminal transactivation domain. These TA-73 isoforms can transactivate $\mathrm{p} 53$ target genes and induce apoptosis. In addition, TA-p73 is induced by a wide variety of chemotherapeutic drugs, such as adriamycin and cisplatin ${ }^{9-12}$ and blocking of TA-p 73 function has been shown to enhance chemoresistance. ${ }^{11,13}$ Other p73 isofoms, namely $\Delta \mathrm{TA}$ 73 , lack the transcriptional activation domain and block the pro-apoptotic function of $\mathrm{p} 53$ and TA-p $73 .{ }^{14,15}$ Thus, overexpression of $\triangle \mathrm{TA}-73$ isoforms can block chemotherapy induced apoptosis in tumor cells that retain wild-type p5 $3 .^{15,16}$

Interestingly, a strong expression of $\mathrm{p} 73$ was found in metastatic melanomas, suggesting that $\mathrm{p} 73$ might function as a positive regulator of tumor growth in the progression from primary melanoma to metastases. ${ }^{17}$ Detailed analyses of $\mathrm{p} 73$ isoforms revealed an overexpression of $\Delta T A-73$ isoforms in metastatic melanomas, suggesting a potential function of $\triangle \mathrm{TA}-\mathrm{p} 73$ in melanoma progression due to inhibition of $\mathrm{p} 53$ and TA-p73 function. ${ }^{18}$

These findings indicate that the ratio of TA-p 73 to $\Delta \mathrm{TA}$ p73 may be an important determinant of drug response. 
Thus, we hypothesized that restoring TA-p73 function by overexpression could confer sensitivity to chemotherapyinduced apoptosis in tumor cells. Here we demonstrate that upregulation of TA-p $73 \beta$ expression by adenoviral gene transfer enhances chemosensitivity to adriamycin and cisplatin in a subset of melanoma cell lines, both in vitro and in vivo. Our data emphasizes the need of a comprehensive analysis of $\mathrm{p} 53$ homologues in melanoma cells to understand the molecular basis of drug resistance in this aggressive neoplasm.

\section{Materials and methods}

\section{Cell lines and culture conditions}

Human melanoma cell lines Mel1 (SK-Mel-19), Mel2 (SK-Mel-29), Mel3 (SK-Mel-103), Mel4 (SK-Mel-147), Mel5 (G-361), Mel6 (UACC-257), all wild-type p53, were derived from metastatic melanomas from patients. Mel1-Mel6 were cultured in DMEM. DMEM was supplemented with $10 \%$ heat-inactivated fetal bovine serum (PAA Laboratories, Coelbe, Germany) and 100 units $/ \mathrm{ml}$ penicillin/streptomycin (GIBCO). Mel7 (WM115; generously provided by Dr. Birgit Schittek; Department of Dermatology, Eberhard-Karls University, Tübingen, Germany), wild-type p53, derived from primary melanoma, was cultured in MCDB. MCDB was supplemented with 20\% Leibowitz cell culture medium (L-15; GIBCO), $2.5 \%$ heat-inactivated FBS, $100 \mathrm{mg} / \mathrm{ml}$ gentamycin (PAA), and $40 \mathrm{U} / \mathrm{ml}$ insulin (Aventis). Cells were cultured in a $5 \%$ $\mathrm{CO}_{2}$ incubator at $37^{\circ} \mathrm{C}$. Medium was changed every 3 days.

\section{Adenoviral vectors}

Three replication-deficient recombinant adenoviral vectors, Ad-GFP, Ad-GFP-TA-p $73 \beta$, and Ad-TA-p $73 \beta$, expressing transgenes under the control of the $\mathrm{CMV}$-promoter have been described previously. ${ }^{19,20}$ All vectors were propagated in 293 cells, purified by $\mathrm{CsCl}$ buoyant density centrifugation and titrated by standard plaque assays.

\section{Chemotherapeutic agents}

For in vitro use adriamycin (ADR, doxorubicin; Pfizer) and -cisplatin (cDDP, [cis-platinum(II)-diammine dichloride]; GRY) were diluted in culture medium before addition to cell cultures. For in vivo use drugs were diluted in $100 \mu \mathrm{l}$ $0.9 \%$ saline immediately before i.p. administration into nude mice.

\section{Adenovirus infection and drug treatment}

$1 \times 10^{6}$ melanoma cells were plated in $10-\mathrm{cm}$ tissue culture plates. After $24 \mathrm{~h}$, cells were infected with adenoviral vector at a multiplicity of infection (MOI 10 to 100) which allows $100 \%$ transduction of each cell line. For combination treatment, chemotherapeutic agents were added to infected cells at a concentration of $0.3 \mu \mathrm{g} / \mathrm{ml}$ for adriamycin and $3 \mu \mathrm{g} / \mathrm{ml}$ for cisplatin. Cells were harvested 3 days after infection.

\section{Real-time RT-PCR}

Total RNA was isolated with the RNeasy Mini Kit (Qiagen) according to the manfactures protocol and $1 \mu \mathrm{g}$ RNA was used for reverse transcription with Omniscript RT (Qiagen) using random hexamers (Applied Biosystems). PCR reactions contained 65 ng cDNA, $1 \times$ Lithos qPCR $^{\text {TM }}$ SYBR Green I Master Mix (Eurogentec), $150 \mathrm{nM}$ of each primer, $3.5 \mathrm{mM} \mathrm{MgCl} 2$ and $0.5 \mathrm{mg} / \mathrm{ml} \mathrm{BSA}$ (Roche) and were carried out in a LightCycler (RocheDiagnostics). Amplification products for TA-p $73, \mathrm{p} 73 \Delta \mathrm{ex} 2, \mathrm{p} 73 \Delta \mathrm{ex} 2 / 3$, $\Delta \mathrm{N}^{\prime}-\mathrm{p} 73, \quad \Delta \mathrm{N}-\mathrm{p} 73, \mathrm{p} 21^{\text {WAF1/CIP1 }}$ and S9 ribosomal protein (S9ribP) were verified by melting curves, agarose gel electrophoresis and direct sequencing. PCR conditions, primer sequences and control plasmids were described previously. ${ }^{14,18}$

\section{Western blotting}

Cells were lysed in RIPA buffer $(50 \mathrm{mM}$ Tris-Cl, $150 \mathrm{mM} \mathrm{NaCl}, 1 \% \mathrm{NP}-40,0.5 \%$ sodium deoxycholate, $0.1 \%$ SDS) and total protein concentration was quantitated by Bradford assay. Equal amounts of each sample were separated by SDS-PAGE, transferred to nitrocellulose membranes (Amersham-Biosciences; Freiburg, Germany) and probed with antibodies against cleaved Caspase-3 (\#9661), full-length PARP (\#9542), or cleaved PARP (\#9541) from Cell Signaling Technology (Frankfurt, Germany). Reprobing of the blot with actin antibody (Santa Cruz Biotechnology; \#sc-1616, Heidelberg, Germany) served as gel loading control. Primary antibodies were detected with appropriate secondary antibodyhorseradish peroxidase conjugates (Amersham Pharmacia, Braunschweig, Germany) according to the enhanced chemiluminescence protocol (Pierce).

\section{Flow cytometry analysis}

For flow cytometry analysis, adherent and nonadherent cells were harvested, fixed in $70 \%(\mathrm{v} / \mathrm{v})$ ethanol and stained for DNA content with propidium iodide. FACS analysis was performed at 3 days after infection. The data from 10,000 cells were collected and analyzed using CellQuest ${ }^{\mathrm{TM}}$ soft- 
ware (Becton Dickinson). The subdiploid population was calculated as an estimate of the apoptotic cell population. Analysis was carried out in a FACSCalibur flow cytometer (Becton Dickinson, San Diego, USA) using CellQuest Software.

\section{Hoechst 33342 staining}

At 3 days post infection Hoechst 33342 was added to the cell culture medium at $1 \mu \mathrm{g} / \mathrm{ml}$. Cells were incubated at $37^{\circ} \mathrm{C} / 5 \% \mathrm{CO}_{2}$ and subsequently subjected to fluorescence microscopy.

\section{TUNEL assay}

The immunohistochemic detection of apoptosis in formalinfixed, paraffin-embedded tumor tissues was performed by using the Promega DeadEnd ${ }^{\mathrm{TM}}$ Fluorometric TUNEL System. The TUNEL reaction mixture $(50 \mu \mathrm{l})$ was applied to deparaffinized and rehydrated tumor sections and the slides were incubated for $60 \mathrm{~min}$ at $37^{\circ} \mathrm{C}$. The tissues were counterstained with propidiumiodide and apoptotic cells were determined by microscopic examination. For each tumor three different slides were examined.

\section{Animal experiments}

Tumors were established by subcutaneous injection of $5 \times 10^{6} \mathrm{Mel} 3$ or Mel4 cells into the right flank of athymic $\mathrm{BALB} / \mathrm{c}$ nu/nu mice (6-8 weeks of age). Mice with visible and palpable tumors received three direct intratumoral injection of purified Ad-TA-p73 $\beta\left(5 \times 10^{9}\right.$ pfu $)$ or AdGFP $\left(5 \times 10^{9}\right.$ pfu $)$ every 3 days. Virus was diluted in total volume of $100 \mu \mathrm{l}$ of $0.9 \%$ saline and administered in a single pass of a 27 -gauge hypodermic needle using constant infusion pressure. Beginning on day 7 , mice were treated with ADR (1 mg/kg), cDDP $(6 \mathrm{mg} / \mathrm{kg})$ or $0.9 \%$ saline via i.p. injections every 3 days, for a total of five treatments. Each experimental group consists of 6 mice. The individual groups received the following treatment combinations: group1, Ad-GFP+0.9\% NaCl; group2, AdGFP+ADR; group3, Ad-GFP+cDDP; group4, Ad-TA$\mathrm{p} 73 \beta+0.9 \% \mathrm{NaCl}$; group5, Ad-TA-p73 $\beta+$ ADR; group6, Ad-TA-p $73 \beta+\mathrm{cDDP}$. Tumors were measured every 3 days in two perpendicular diameters using calipers, and tumor size was calculated using the formula: length $(\mathrm{mm}) \times$ width $\left(\mathrm{mm}^{2}\right) / 2$. Animals were sacrificed when the longest diameter was greater than $15 \mathrm{~mm}$ or when any two measurements were greater than $10 \mathrm{~mm}$. Animal experiments were performed in accordance with institutional guidelines set by the University Institutional Animal Care and Use Committee.

\section{Statistical analysis}

Statistic analysis was performed using paired two-sided Student's $t$ test. $P<0.05$ was considered as statistically significant.

\section{Results}

\section{p73 expression status in melanoma cell lines}

In order to determine which $\mathrm{p} 73$ isoforms are expressed in the melanoma cell lines, total cellular RNA was purified from cultured melanoma cells and transcript levels of TAp73 and $\Delta$ TA-p73 (p73 $\Delta$ ex2, p73 $\Delta 2 / 3, \Delta \mathrm{N}^{\prime}-\mathrm{p} 73, \Delta \mathrm{N}-$ p73; Figure 1A) were quantified using real-time RT PCR. As shown in Figure 1B, TA-p73 expression was observed in all melanoma cell lines with highest transcript levels in Mel7 and lowest transcript levels in Mel3. $\triangle$ TA-p73 isoforms $\mathrm{p} 73 \Delta \mathrm{ex} 2, \mathrm{p} 73 \Delta 2 / 3$ and $\Delta \mathrm{N}$-p 73 were expressed in all melanoma cell lines, except in $\mathrm{Mel} 3$ (p73 $\Delta \mathrm{ex} 2$ and $\Delta \mathrm{N}$ p73 mRNA not detectable). In contrast, $\Delta N^{\prime}-\mathrm{p} 73$ transcript expression was only detectable in $3 / 7$ melanoma cell lines (Mel2, Mel6, Mel7). Thus, all melanoma cell lines significantly expressed both proapoptotic TA-p73 and antiapoptotic $\triangle T A-p 73$ transcripts. An important issue to consider is the ratio of the two antagonistic p 73 protein classes. 5/7 melanoma cell lines showed an overweight of $\triangle T A-p 73$ transcripts ( $\triangle \mathrm{TA}-\mathrm{p} 73 / \mathrm{TA}-\mathrm{p} 73$ ratios: $\mathrm{Mel} 5$, 6.9; Mel1, 5.4; Mel4, 4.2; Mel7, 2.3; Mel2, 2.1), whereas 2/7 melanoma cell lines revealed an overexpression of TA-p 73 ( $\triangle \mathrm{TA}-\mathrm{p} 73 / \mathrm{TA}-$ p73 ratios: $\mathrm{Mel3}, 0.8 ; \mathrm{Mel} 6,0.4)$. On the protein level the $\triangle$ TA-p73/TA-p73 ratio might be higher, because the $\Delta T A-$ p73 proteins were reported to be more stable. ${ }^{19}$ However, at present, it is not known how much $\triangle \mathrm{TA}-\mathrm{p} 73$ activity is required to outweigh the tumor suppressor function of TA-p73. Taken together these data support a possible oncogenic function of $\triangle T A-p 73$ in the investigated melanoma cell lines.

\section{Induction of $\mathrm{p} 21^{\mathrm{WAF} 1 / \mathrm{Cip} 1}$ by TA-p73 $\beta$ in melanoma cell lines}

First, functional activity of the Ad-GFP-TA-p73 $\beta$ vector was tested in melanoma cell lines by measuring expression of p73 and its downstream target $\mathrm{p} 21^{\mathrm{WAF} 1 / \mathrm{Cip} 1}$. Cells were infected either with Ad-GFP-TA-p73 $\beta$ or Ad-GFP, and transcripts levels of TA-p 73 and p21 WAF1/Cip1 were quantified at $36 \mathrm{~h}$ after infection using real-time RT PCR (Figure 2). As suggested, Ad-GFP-TA-p $73 \beta$ infected melanoma cell lines showed increased TA-p73 expression in comparison to untreated controls with a fold induction ranging from 3.9 (Mel2) to 13.3 ( $\mathrm{Mel} 5$ ), whereas upregulation of TA-p73 was not observed in Ad-GFP treated melanoma cells. In all melanoma cell lines, p21 WAF1/Cip1, known to be a p73dependent gene, was significantly transactivated following 
Figure 1. Expression of $\mathbf{N}$-terminally truncated TP73 transcript isoforms in melanoma cell lines. (A) Schematic diagram of the various $\triangle T A-$ p73 isoforms. Exon structure of individual mRNAs, the promoter, from which they are transcribed, the encoded protein and their transactivation function are as indicated. Red, transactivation domain; orange, exon 3B-derived coding sequence; blue, DNA-binding domain; grey, unique 5' untranslated region of the $\Delta \mathrm{N}$-p73 transcript. Primer localization is marked by arrows. (B) TP73 transcripts (TA-p73, p73 $\Delta$ ex2, p73 $\Delta 2 / 3$, $\Delta \mathrm{N}^{\prime}$-p73, $\Delta \mathrm{N}$-p73) were quantified by real-time PCR. The bars show the absolute copy number normalized to S9 ribosomal protein (S9ribP) transcript levels as a housekeeping gene. Errors bars indicate one SD. n.s. transcript expression not significant $(P$-values $<0.05$ were regarded as statistically significant; paired, two-sided $t$-test).

\begin{tabular}{|c|c|c|c|c|}
\hline thPHA & & prenoter & prot it & taisactlatsis \\
\hline TAp73 & 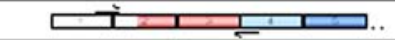 & \multirow{4}{*}{ TA } & TA, 73 & $+(\pi A)$ \\
\hline p7 3बe $x 2$ & 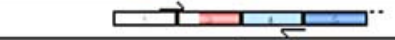 & & p73sexz & \multirow{4}{*}{$-(\Delta T A)$} \\
\hline p7 $348 \times 20$ & 닫. & & p7 $340 \times 23$ & \\
\hline$\Delta H-p 73$ & 1 & & \multirow{2}{*}{$\Delta H-p 73$} & \\
\hline$\Delta 14 p 73$ & ᄅ" & $\Delta H$ & & \\
\hline
\end{tabular}

\section{$\mathrm{B}$}

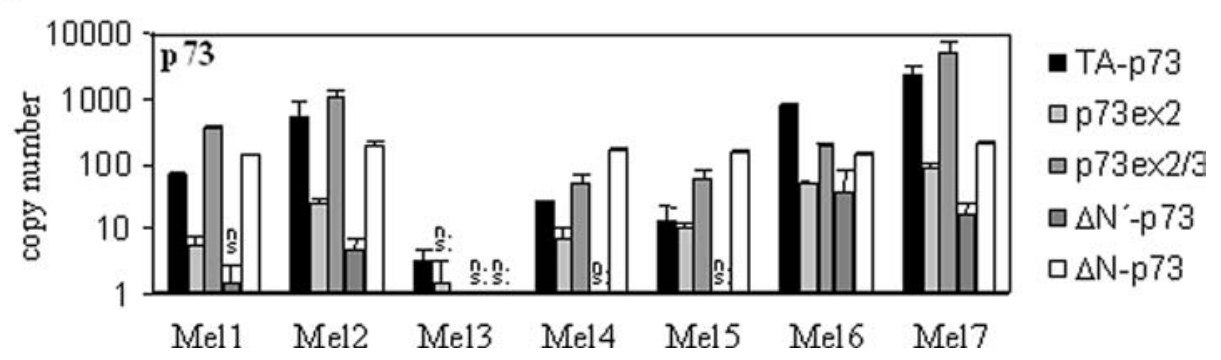

Figure 2. Expression of TA-p73 (black bars) and p21 (gray bars) transcripts in melanoma cells $36 \mathrm{~h}$ after infection with Ad-GFP-TA-p73 $\beta$ or Ad-GFP. Transcript copy numbers are calculated as fold inductions to untreated controls. Error bars indicate one SD of two independent experiments. *, Significant differences $(P<0.05$; paired, two sided $t$-test) between Ad-GFP and Ad-GFP-TA-p73 $\beta$ infected cells.

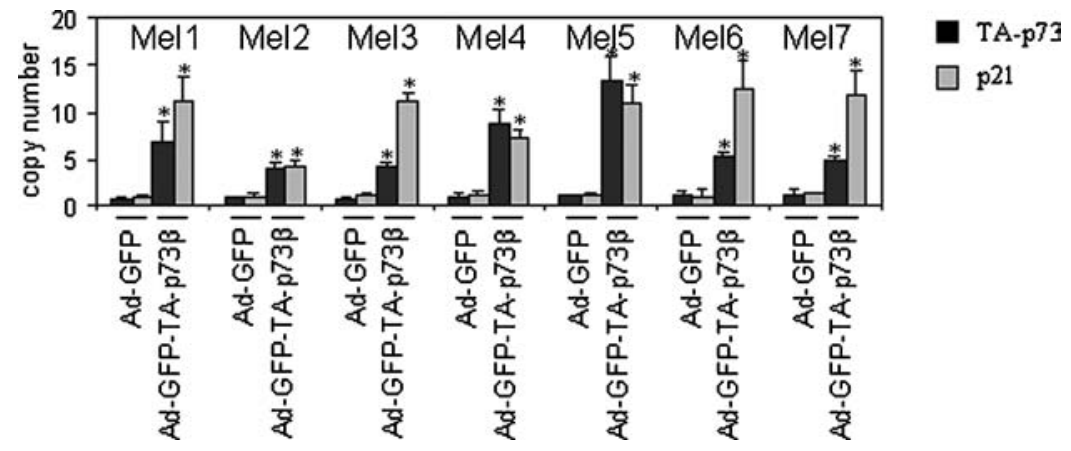

Ad-GFP-TA-p73 $\beta$ infection resulting in a $4.1-(\mathrm{Mel} 2)$ to 12.6-fold (Mel6) induction. In contrast, Ad-GFP infection did not contribute to higher $\mathrm{p} 21^{\mathrm{WAF} 1 / \mathrm{Cip} 1}$ transcript levels.

\section{Induction of apoptosis by TA-p73 $\beta$ in melanoma cell lines}

In order to examine whether apoptosis was induced by the TA-p $73 \beta$ protein, melanoma cell lines were infected either with Ad-GFP-TA-p73 $\beta$ or control virus (Figure 3). The fate of infected GFP-positive melanoma cells was followed by fluorescence microscopy. Unlike Ad-GFP infected cells which had a normal morphology (Figure 3A), cell overexpressing TA-p $73 \beta$ rounded up and showed typical features of apoptotic cells characterized by membrane blebbing, cell shrinkage, and intense staining of condensed chromatin as analyzed by Hoechst 33342 staining (Figure 3B). As demonstrated in Figure $3 \mathrm{C}$, increased expression of TA$\mathrm{p} 73 \beta$ in melanoma cells (shown for Mel4) was accompanied by caspase-3 (Casp-3) activation and by cleavage of the $116 \mathrm{kD}$ intact form of PARP to the $89 \mathrm{kD}$ fragment which is intimately linked to the induction of apoptosis. Both Casp-3 and PARP cleavage became evident as early as $72 \mathrm{~h}$ after infection. Flow cytometry analysis of cells infected with Ad-GFP-TA-p $73 \beta$ revealed a sub-G1 population indicative 
Figure 3. Induction of apoptosis in melanoma cells by adenoviral TA-p73 $\beta$ transfer. Mel4 is shown as a representative cell line. (A) Cells were stained with Hoechst 33342 at $72 \mathrm{~h}$ after infection and analyzed by laser scan microscopy. Infected cells are indicated as GFP positive. (B) Higher magnification (x 20) of Ad-GFP-TA-p73ß infected cells showing typical features of apoptotic cells with membrane blebbing, cell shrinkage, and nuclear condensation. (C) Caspase-3 activation and PARP cleavage analyzed by Western blotting using antibodies detecting the 19 and $17 \mathrm{kD}$ subunits of cleaved caspase-3 (Casp-3), full-length PARP and the $89 \mathrm{kD}$ proteolytic cleavage product. Actin was used as a loading control.
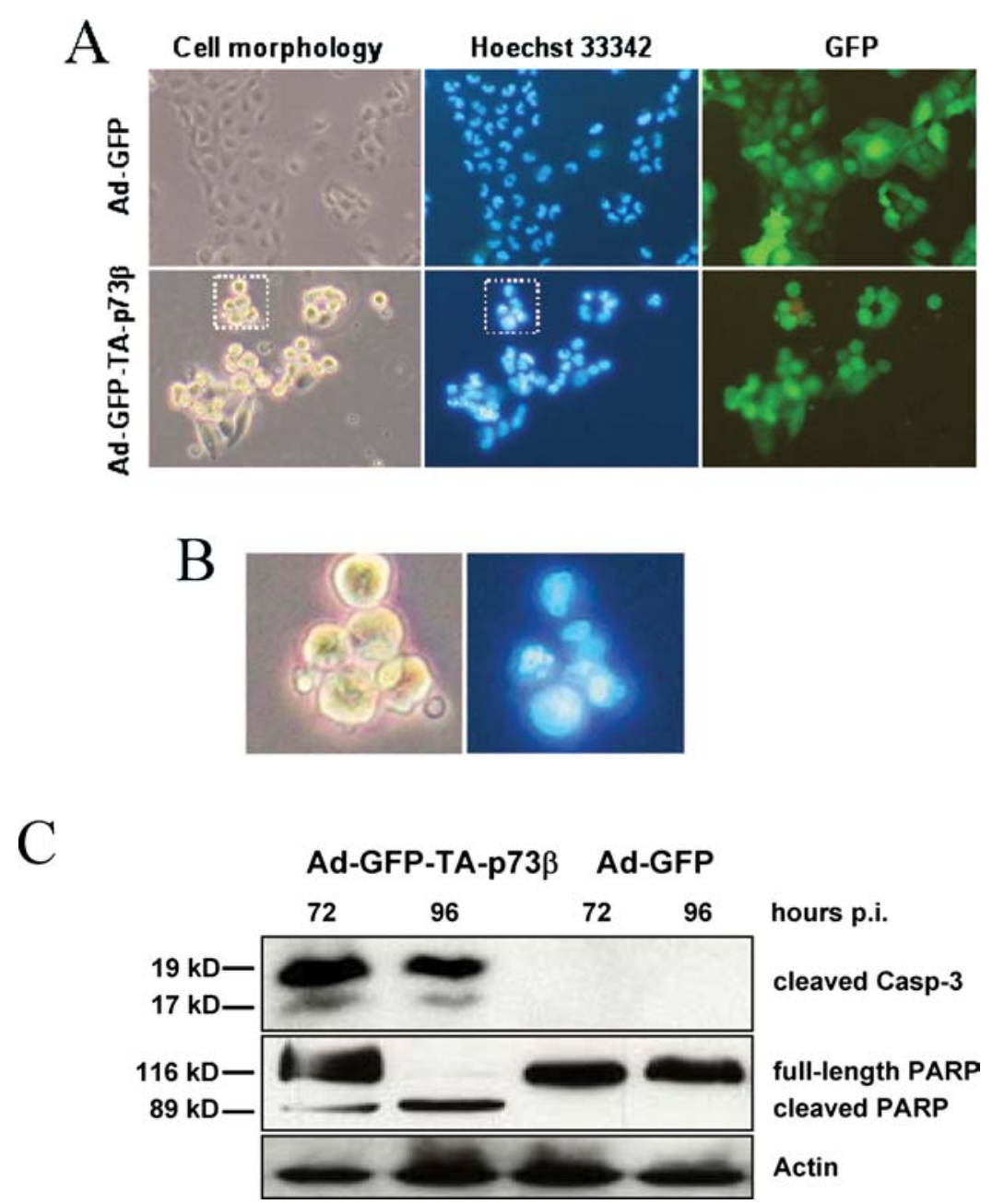

for apoptosis ranging from $7.7 \%$ (Mel6), 17.4\% (Mel7), 20.6\% (Mel4), 32.5\% (Mel1), 36.6\% (Mel2), 36.5\% (Mel3) to $42.1 \%(\mathrm{Mel} 5)$ at 3 days after infection (Figure 4$)$, indicating that the capability of TA-p73 $\beta$ to induce apoptosis strongly differs between individual melanoma cell lines.

\section{Sensitization of melanoma cell lines to adriamycin and cisplatin by Ad-mediated TA-p73 $\beta$ gene transfer in vitro}

Recent reports showed that endogenous TA-p73 cooperates with DNA-damaging agents to induce apoptosis in tumor cells. $^{9-12}$ Next, we investigated whether increased levels of exogenous TA-p $73 \beta$ in melanoma cells could enhance their sensitivity to chemotherapeutic drugs. Melanoma cells infected with adenovirus vector (Ad-GFP-TA-p73 $\beta$ or Ad-GFP) were subsequently treated with adriamycin or cisplatin and harvested for cell cycle analysis. Ad-GFP-TA$\mathrm{p} 73 \beta$ infection resulted in a marked sensitization of several melanoma cell lines to low concentrations of adriamycin $(A D R)$ and cisplatin $(c D D P)$ as measured by flow cytometry (see Figure 4A for an example of Mel4 and Figure $4 \mathrm{~B}$ for quantification analyses with all the cell lines in this study).

In lines 6 and 7 , the effect of TA-p $73 \beta$ showed an additive effect with ADR or cDDP (Figure $4 \mathrm{~B}$ ). In Lines 1, 2 and 5 , the impact of TA-p73 $\beta$ on drug response was synergistic with both drugs, and in lines 3 and 4 with ADR. For example, compare the $12 \%$ or $18 \%$ killing by ADR or cDDP single agents, respectively with a $80.6 \%$ in the presence of overexpressed TA-p73 $\beta$ (-note that killing with TA-p $73 \beta$ is limited to $32.5 \%$; see Figure $4 \mathrm{~B}$ ). In contrast, Ad-GFP infection had no obvious effect on drug sensitivity. Taken together, these data indicate that adriamycin and/or 
Figure 4. Apoptotic effect of Ad-GFP-TA-p73ß and adriamycin or cisplatin combination treatment on human melanoma cells. Melanoma cells were treated with Ad-GFP, Ad-GFP-TA-p73B, adriamycin $(A D R)$, cisplatin $(c D D P)$ or combinations thereof as shown. Chemotherapeutic agents were used at a concentration of $0.3 \mu \mathrm{g} / \mathrm{ml}$ for ADR and $3 \mu \mathrm{g} / \mathrm{ml}$ for cDDP. (A) Flow cytometry profiles showing DNA content (x-axis) against cell number ( $y$-axis). Mel4 is shown as a representative sample. The percentage of cells with a sub-G1 DNA content is as indicated. (B) Flow cytometry results of two independent experiments \pm SD (control light gray, Ad-GFP infected dark gray, Ad-GFP-TA-p73 $\beta$ infected black). * Significant differences $(P<0.05$ paired, two sided $t$ test) between Ad-GFP-TA-p73 $\beta$ and Ad-GFP infected cells or control cells.
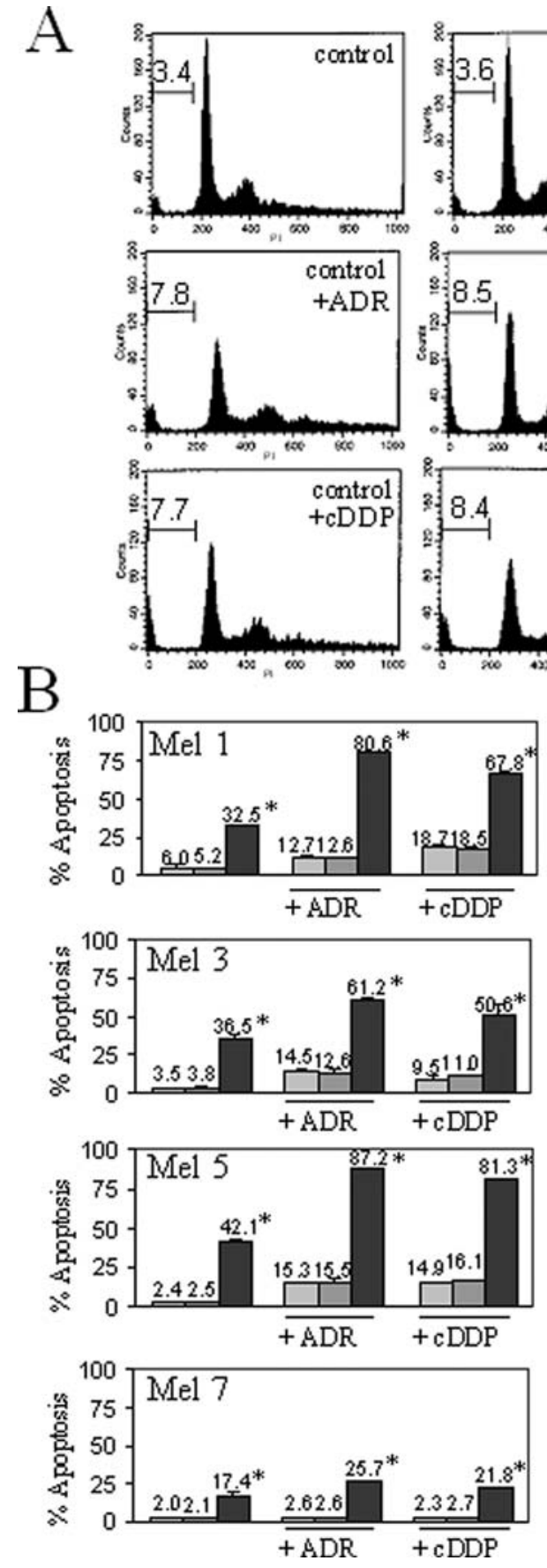
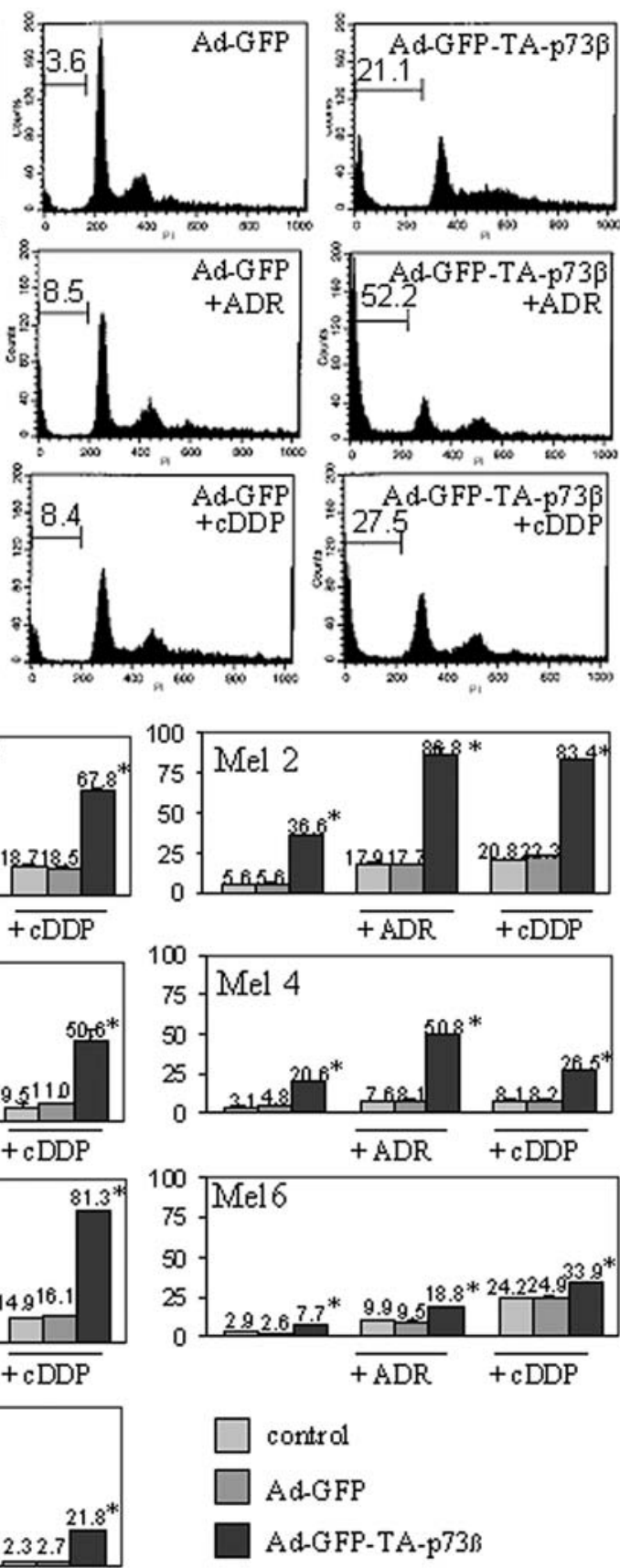

cisplatin mediated cell killing can be efficiently enhanced by TA-p $73 \beta$ gene expression in the majority of melanoma cell lines. Apoptosis induction in response to Ad-GFP-TA$\mathrm{p} 73 \beta+\mathrm{ADR}$ and Ad-GFP-TA-p $73 \beta+\mathrm{cDDP}$ combination therapies was also confirmed by Hoechst 33342 staining (data not shown).

\section{Antitumor effect of TA-p73 $\beta$ and ADR/cDDP combination therapy in vivo}

To determine the physiological impact of the results we obtained in tissue culture systems, we analyzed the therapeutic efficacy of combination therapy on growing tumors 
Figure 5 (A) In vivo antitumor effect of TA-p73 $\beta /$ ADR or TA-p73 $\beta /$ cDDP combination therapy. After s.c. administration of $5 \times 10^{6}$ Mel3 or Mel4 cells, palpable tumors were randomized to receive a local injection of Ad-GFP or Ad-TA-p73 $\beta$ on days 6,9 and 12 followed by i.p. injection of ADR $(1 \mathrm{mg} / \mathrm{kg})$ or $\operatorname{cDDP}(6 \mathrm{mg} / \mathrm{kg})$ on days $7,10,13,16,19$. The total virus dose was $3 \times 5 \times 10^{9}$ pfu. Mice were monitored for tumor regression over 21 days. The results are expressed as the mean from each treatment group \pm SD. The differences in tumor size in animals treated with Ad-TA-p73 $\beta+$ ADR or Ad-TA-p73 $\beta+$ CDDP were statistically significant compared with mice treated with Ad-TA-p73 $\beta$ alone, Ad-GFP alone, Ad-GFP+ ADR or Ad-GFP+CDDP (day21, $P<0.05$; paired two-sided $t$-test). The differences in tumor size in animals treated with Ad-TA-p73 $\beta$ alone were statistically significant compared with mice treated with Ad-GFP alone, Ad-GFP+ADR or Ad-GFP+cDDP (day21, $P<0.05$; paired, two-sided $t$-test). (B) TUNEL staining for apoptotic cells in melanoma tissues (representative Mel3 tumors are shown) at $72 \mathrm{~h}$ after intratumoral injection of $5 \times 10^{9} \mathrm{pfu}$ Ad-GFP or Ad-TA-p73 $\beta$. Increased apoptotis (green cells) was detected in Ad-TA-p73 $\beta$ treated tumor tissue. A higher magnification $(x 20)$ of apoptotic cells is shown in the right panel.
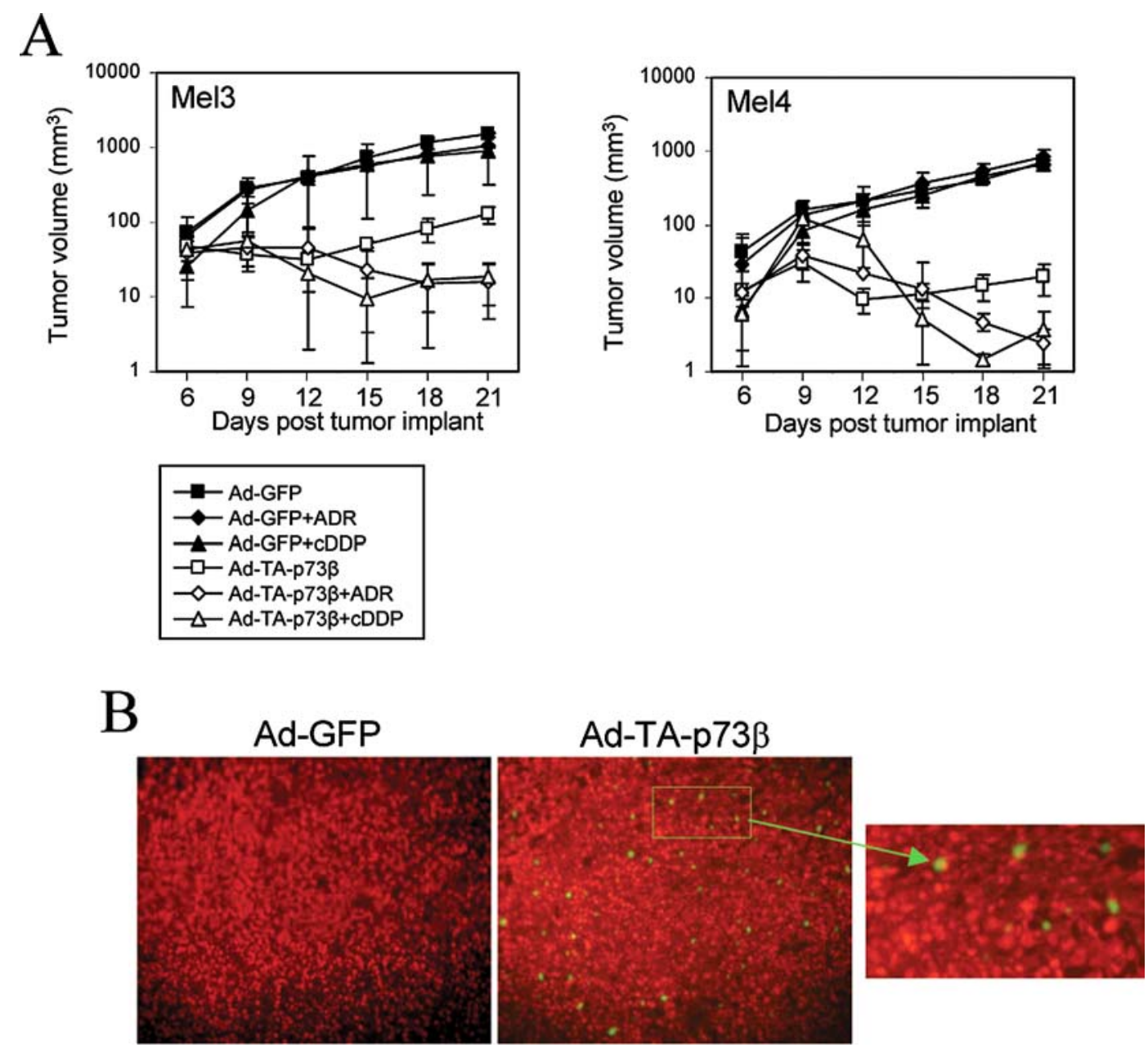

in mice. Established subcutaneous Mel3 and Mel4 tumors were treated by three intratumoral injections of Ad-GFP or Ad-TA-73 $\beta$ followed by five i.p. injections of either ADR or cDDP over a period of 21 days and monitored for regression (Figure 5A). Both Ad-GFP alone and in combination with either drug showed a similar effect resulting in a pronounced tumor growth. Compared to the controls, tumor growth was markedly delayed after injection of Ad-TA-p $73 \beta$ (day 21, $P<0.05$; paired two-sided $t$-test). In both tumor models, a significant reduction of tumor size was observed only after combination treatment of Ad-TA-p $73 \beta$ and adriamycin or cisplatin (day 21, $P<0.05$; paired two-sided $t$-test; Figure 5), which resulted in a three week longer survival of mice (data not shown). Importantly, no signs of systemic toxicity were observed in animals receiving the combina- tion therapy. These findings are in accordance to our in vitro data, which demonstrated a synergistic effect of combined Ad-TA-p73 $\beta /$ ADR treatment and an additive effect of AdTA-p $73 \beta / \mathrm{cDDP}$ combination therapy on cell death in Mel3 and Mel4 cells (Figure 4). Compared to Ad-GFP treated controls, a positive TUNEL staining was detected only in melanoma tissue injected with Ad-TA-p73 $\beta$ (Figure 5B), indicating that apoptosis induction is the mechanism of p73-mediated cytotoxicity in vivo.

\section{Discussion}

The success of systemic therapy for metastatic melanomas has been minimal. Chemotherapeutic drugs could theoretically target metastatic melanomas, but current treatments 
do not provide a significant therapeutic benefit. ${ }^{1}$ One of the major mechanisms that contribute to chemotherapy mediated tumor cell death is the induction of apoptosis. The extreme resistance of malignant melanoma to chemotherapeutic drugs, and the fact that apoptotic indices are typically low in melanoma tumors, particularly at advanced stages, ${ }^{21}$ prompted an intense search for acquired apoptotic defects during melanoma progression. ${ }^{2}$

p53 plays a central role in the apoptotic response to DNA damaging agents. Consistent with its role in chemotherapy induced apoptosis, p53 mutations have been linked to chemoresistance in many types of cancer. Nonetheless, melanomas display a low frequency of p53 mutations. ${ }^{6}$ One explanation for the extreme chemoresistance in the presence of wild-type p53 in melanomas is that upstream p53 regulators or downstream p53 mediators may functionally replace p53 loss during melanomagenesis. For example Apaf- 1 , which is an effective downstream mediator of p53-induced apoptosis, is frequently downregulated in metastatic cell lines and tumor specimens ${ }^{22-24}$ and restoration of Apaf-1 function through gene transfer has been shown to enhance chemosensitivity. ${ }^{4}$ However, other mechanisms should contribute to the malignant phenotype of melanoam cells since Apaf-1 overexpression does not completely restore drug response. ${ }^{25}$ We had previously shown that metastatic melanoma cells have a dysregulated expression of different isoforms of $\mathrm{p} 73$, a factor that shares tumor suppressor functions with $\mathrm{p} 53$. Here we show that melanoma cells express a higher ratio of anti- to pro-apoptotic isoforms of $\mathrm{p} 73$. Reverting this ratio by overexpression of fulllength $\mathrm{p} 73$ significantly sensitized melanoma cells to the the chemotherapeutic drugs adriamycin and cisplatin (otherwise poorly effective in melanoma).

It has been puzzling that $\mathrm{p} 73$, to the contrary to $\mathrm{p} 53$, can be upregulated in human tumors. In some cases, overexpression of p73 could even be correlated with an advanced tumor stage or poor prognostic parameters. ${ }^{26,27}$ Moreover, various chemotherapeutic drugs, such as adriamycin and cisplatin, mediate apoptosis in cancer cells in part by upregulating p 73 transcripts and stabilizating p 73 protein via c-Abl. ${ }^{9-12}$ However, the fact that anti-apoptotic isoforms can also be upregulated in tumor cells, $8,14,15$ emphasizes the complexity of $\mathrm{p} 73$ function. The $\Delta \mathrm{TA}-\mathrm{p} 73$ proteins can be generated by the following two mechanisms: (a) abberant splicing (p73 $\Delta \mathrm{ex} 2, \mathrm{p} 73 \Delta \mathrm{ex} 2 / 3, \Delta \mathrm{N}^{\prime}$-p 73 ) and (b) alternative usage of a second intrinsic promoter $(\Delta \mathrm{N}-\mathrm{p} 73) .{ }^{8} \Delta \mathrm{TA}-\mathrm{p} 73$ proteins were shown to compromise p53- and TA-p73-dependent apoptosis ${ }^{16,19}$ and thereby to promote malignant transformation. ${ }^{15,28}$

Malignant melanomas have also been shown to express high levels of wild-type p $73,{ }^{17,29}$ but also $\Delta$ TA-p 73 levels (mostly TA-promoter derived $\mathrm{p} 73 \Delta \mathrm{ex} 2 / 3$ and $\mathrm{p} 73 \Delta \mathrm{ex} 2$ isoforms). ${ }^{18}$ Thus, $\Delta$ TA-p73 upregulation is another possible mechanism that may functionally replace $\mathrm{p} 53$ loss during melanomagenesis. Here we further extend this previous data, showing that transactivation-deficient, anti-apoptotic $\triangle T A-p 73$ isoforms are expressed in $7 / 7$ of our melanoma cell lines, suggesting a possible role of $\triangle T A-p 73$ in apoptosis-deficiency and chemoresistance by inhibition of $\mathrm{p} 53$ and TA-p 73 function.

Thus, we hypothesized that a therapeutic approach for sensitizing melanoma cells to chemotherapeutic drugs could be antagonizing $\triangle \mathrm{TA}-\mathrm{p} 73$ function and restoring p73-mediated apoptosis by overexpression of TA-p73. In melanoma cell lines both $\mathrm{p} 53$ and TA-p $73 \beta$ gene transfer has been reported to promote apoptosis. ${ }^{30,31}$ Here we show that TA-p $73 \beta$ is more effective as a chemosensitizing factor. When TA-p $73 \beta$ was combined with adriamycin we observed a synergistic effect on apoptotic cell death in 5/7 melanoma cell lines in vitro. As a potential reason of why these five cell lines, and not the other two, are sensitive to TA-p $73 \beta$ one could expect differences in the ratio of $\triangle$ TA:TA isoforms. However, there was no significant correlation between the lack of TA-p $73 \beta$-mediated chemosensitivity and the ratio of anti-apoptotic versus apoptotic p73 in these cell lines. We can thus speculate that other factors such as a variable induction of $\mathrm{p} 73$ downstream target genes or the failure of some cells to express the transduced gene even after $100 \%$ infection explain the difference in therapeutic outcome. Both, adriamycin and cisplatin also cooperated with TA-p $73 \beta$ to enhance antitumor activity in xenograft tumors using Mel3 and Mel 4 cells. Most importantly, the significant drug-sensitizing effect of TA-p $73 \beta$ found in vivo, supports the development of novel therapeutic strategies aiming to block $\Delta \mathrm{TA}-\mathrm{p} 73$ or to exploit the TA-p73 $\beta$ levels that melanoma cells intrinsically express.

\section{Acknowledgments}

The authors thank Birgit Schittek (Department of Dermatology, Eberhard-Karls University, Tübingen, Germany) for providing the WM115 melanoma cell line and Arndt Rolfs (Department of Neurology, University of Rostock, Germany) for providing the LightCycler. This work was supported by grant 10-1934-Pü3 from the Deutsche Krebshilfe, Dr. Mildred Scheel Stiftung and FORUN.

\section{References}

1. Bajetta E, Del Vecchio M, Bernard-Marty C, et al. Metastatic melanoma: chemotherapy. Semin Oncol. 2002; 29: 427-445.

2. Soengas MS, Lowe SW. Apoptosis and melanoma chemoresistance. Oncogene 2003; 22: 3138-3151.

3. Grossman D, McNiff JM, Li F, Altieri DC. Expression and targeting of the apoptosis inhibitor, survivin, in human melanoma. J. Invest. Dermatol. 1999; 113: 1076-1081.

4. Soengas MS, Capodieci P, Polsky D, et al. Inactivation of the apoptosis effector Apaf-1 in malignant melanoma. Nature 2001; 409: 207-211.

5. Davies H, Bignell GR, Cox C, et al. Mutations of the BRAF gene in human cancer. Nature 2002; 417: 949-954. 
6. Lubbe J, Reichel M, Burg G, Kleihues P. Absence of p53 gene mutations in cutaneous melanoma. J. Invest. Dermatol. 1994; 102 : 819-821.

7. Chin L, Pomerantz J, Polsky D, et al. Cooperative effects of INK4a and ras in melanoma susceptibility in vivo. Genes. Dev. 1997; 11: 2822-2834.

8. Stiewe T, Putzer BM. Role of p73 in malignancy: tumor suppressor or oncogene? Cell Death Differ. 2002; 9: 237245.

9. Gong JG, Costanzo A, Yang HQ, et al. The tyrosine kinase c-Abl regulates $\mathrm{p} 73$ in apoptotic response to cisplatin-induced DNA damage. Nature 1999; 399: 806-809.

10. Yuan ZM, Shioya H, Ishiko T, et al. p73 is regulated by tyrosine kinase c-Abl in the apoptotic response to DNA damage. Nature 1999; 399: 814-817.

11. Irwin MS, Kondo K, Marin MC, Cheng LS, Hahn WC, Kaelin WG Jr. Chemosensitivity linked to p73 function. Cancer Cell 2003; 3: 403-410.

12. Chen X, Zheng Y, Zhu J, Jiang J, Wang J. p73 is transcriptionally regulated by DNA damage, p53, and p73. Oncogene 2001; 20: 769-774.

13. Bergamaschi D, Gasco M, Hiller L, et al. p53 polymorphism influences response in cancer chemotherapy via modulation of p73-dependent aoptosis. Cancer Cell 2003; 3: 387-402.

14. Stiewe T, Zimmermann S, Frilling A, Esche H, Putzer BM. Transactivation-deficient DeltaTA-p73 acts as an oncogene. Cancer Res. 2002; 62: 3598-3602.

15. Zaika AI, Slade N, Erster SH, et al. DeltaNp73, a dominantnegative inhibitor of wild-type p53 and TAp73, is up-regulated in human tumors. J. Exp. Med. 2002; 196: 765-780.

16. Vossio S, Palescandolo E, Pediconi N, et al. DN-p73 is activated after DNA damage in a p53-dependent manner to regulate p53-induced cell cycle arrest. Oncogene 2002; 21: 3796-3803.

17. Zhang H, Schneider J, Rosdahl I. Expression of p16, p27, p53, p73 and Nup88 proteins in matched primary and metastatic melanoma cells. Int. J. Oncol. 2002; 21: 43-48.

18. Tuve S, Wagner SN, Schittek B, Putzer BM. Alterations of DeltaTA-p73 splice transcripts during melanoma development and progression. Int. J. Cancer 2004; 108: 162-166.

19. Stiewe T, Theseling CC, Putzer BM. Transactivation-deficient Delta TA-p73 inhibits p53 by direct competition for DNA binding: implications for tumorigenesis. J. Biol. Chem. 2002; 277: 14177-14185.

20. Rödicker F, Pützer BM. p73 is effective in p53-null pancreatic cancer cells resistant to wild-type TP53 gene replacement. Cancer Res. 2003; 63: 2737-2741.

21. Glinsky GV, Glinsky VV, Ivanova AB, Hueser CJ. Apoptosis and metastasis: increased apoptosis resistance of metastatic cancer cells is associated with the profound deficiency of apoptosis execution mechanisms. Cancer Lett. 1997; 115: 185-193.

22. Fujimoto A, Takeuchi H, Taback B, et al. Allelic Imbalance of 12q22-23 associated with Apaf-1 locus correlates with poor disease outcome in cutaneous melanoma. Cancer Res. 2004; 64: 2245-2250.

23. Dai DL, Martinka M, Bush JA, Li G. Reduced Apaf-1 expression in human cutaneous melanomas. Br. J. Cancer 2004; 91: 1089-1095.

24. Baldi A, Santini D, Russo P, et al. Analysis of APAF-1 expression in human cutaneous melanoma progression. Experiment. Dermatol. 2004; 13: 93-97.

25. Soengas MS, Capodieci P, Polsky D, et al. Inactivation of the apoptosis effector Apaf-1 in malignant melanoma. Nature 2001; 409: 207-211.

26. Sun XF. p73 overexpression is a prognostic factor in patients with colorectal adenocarcinoma. Clin. Cancer Res. 2002; 8: 165-170.

27. Tannapfel A, Wasner M, Krause K, et al. Expression of p73 and its relation to histopathology and prognosis in hepatocellular carcinoma. J. Natl. Cancer Inst. 1999; 91: 1154-1158.

28. Petrenko O, Zaika A, Moll UM. deltaNp73 facilitates cell immortalization and cooperates with oncogenic Ras in cellular transformation in vivo. Mol. Cell Biol. 2003; 23: 5540-5555.

29. Kroiss MM, Bosserhoff AK, Vogt T, et al. Loss of expression or mutations in the $\mathrm{p} 73$ tumour suppressor gene are not involved in the pathogenesis of malignant melanomas. Melanoma Res. 1998; 8: 504-509.

30. Yamashita $\mathrm{T}$, Tokino $\mathrm{T}$, Tonoki $\mathrm{H}$, et al. Induction of apoptosis in melanoma cell lines by $\mathrm{p} 53$ and its related proteins. J. Invest. Dermatol. 2001; 117: 914-919.

31. Sauter ER, Takemoto R, Litwin S, Herlyn M. p53 alone or in combination with antisense cyclin D1 induces apoptosis and reduces tumor size in human melanoma. Cancer Gene Ther. 2002; 9: 807-912. 